\title{
The Development of China 's Over - Seven - holes Hulusi Music Hongfei Liu
}

\author{
School of Music, Hubei Engineering University, Xiaogan Hubei, 432000,China
}

Key words: Over seven holes Hulusi, Hulusi music, Li Chunhua.

\begin{abstract}
In this paper, the advantages and disadvantages of Over seven holes Hulusi and the traditional Hulusi were compared and analyzed. The basic conclusion of the analysis of the results, the present situation and the development potential of the super-seven-hole Hulusi is that the super-seven-hole Hulusi is still in the primary stage of development, and there are contradictions in the process of developing communication with the traditional Hulusi, But the development of Over seven holes Hulusi is the direction of the development of Hulusi, and the traditional Hulusi is also the basis of Over seven holes Hulusi, in the development process of Over seven holes Hulusi to learn from the traditional Hulusi excellent development experience.
\end{abstract}

\section{Over seven holes Hulusi instrument}

Hulusi music in recent years in the social music has been widely disseminated, showing a hot situation, Hulusi music performance theme is also increasingly rich, but the traditional Hulu instrument because of its sound field performance and other defects, has been in the gourd Silk music development process reflects a larger problem. In order to make Hercules music to sustainable development, in recent years, many people in the Hulusi industry began to improve the traditional gourd instruments. Such as: the 20th century, 70 years Hulusi artist Yu Tianyou according to their own playing experience, and proposed and Hulusi Craftsman Liu Zhen jointly developed a double master tube, four pipe, six pipe gourds, and in the reform of the Hulusi On the basis of the key to the test, which created a 17-degree, six-tube tone gourd silk. "Hulusi for the broadening of the range of ideas put forward a new idea; in the last century, the father of Hulusi all proposed Broaden the idea of the gourd range, made in the traditional seven hole Hulusi on the basis of a hole with a hole to increase the range of Hulusi, so that the performance of the eight hole Hulusi was significantly enhanced; and later people have to create a key hoist Silk, nine hole Hulusi, ten hole Hulusi, etc. These practices are reflected in the following methods.

One is by increasing the main sound tube to expand the range, is in a gourd on the two main tube, according to the principle of addition, the composition of the wider range of gourds. The principle of the transformation of reference Xun, Tao flute, there are two reeds, two mouthpiece, playing the song with a different soundtrack playing a different range. But in the practice of playing the process, there are two problems, one is playing in the song if the speed is slightly faster, then the process of switching in the double will be more trouble is not easy to grasp, will also affect the fluency of playing. Second, Hulusi reed each one is not the same, in the process of playing the switch will also affect the performance of the integrity.

One is through the addition of keys to expand the range, refers to the hook through the hook on the sound hole and use the button to control the transformation of the principle of reference to the Western flute flute, saxophone, but the key to make the "super seven hole gourd Silk "without the cooperation between the fingers and the sound hole, it will be in the performance can not reflect the characteristics of Hulusi portamento, sound and so many of the gourd own skills, although the expansion of the range, but lost the unique charm of Hulusi, So there is no spread in the community. 
One is in the gourd sound tube on the hole, the transformation of the reference principle for the Tao flute, the general sound of the instrument in the sound of the more sound the more the sound so the more the range of the wider, generally eight holes, nine holes and ten holes Hulusi. Compared to the previous two expansion of the scope of the transformation of the Hulusi, plus holes can retain the charm of their own gourd, playing will not appear to switch, but the tone of different problems. Hulusi sound principle is through the blowing of the reeds to produce vibration, in the production of this hole will be a large number of Hulusi reed put forward a lot of technical requirements.

"Over seven holes Hulusi" is made by this method of adding holes, it refers to the traditional Hulusi-based single tube, without the key more than seven holes of the gourd instruments collectively, including Eight hole Hulusi, nine hole Hulusi, ten hole Hulusi and so on. Although the "super-seven hole Hulusi" Hulusi reed because the technology is not mature enough reason has not been widely popular, but still by a lot of Hulusi artists and enthusiasts respected, after a large number of Hercules craftsmen continuous improvement Now the eight holes, nine hole gourd reed and the overall performance tends to improve. As the impact of the process of communication in the larger, so now the community is generally called "Over seven holes Hulusi" refers to the hole of the eight holes, nine hole Hulusi. Technology, the production cost will be correspondingly improved, and now both the network sales or physical sales, the general stability of the "Over seven holes Hulusi" pricing in the general seven hole Hulusi 4 to 5 times. This has become a "Over seven holes Hulusi" no large area to promote the dissemination of a factor.

\section{Over - Seven - holes Hulusi Music}

\section{Creation of Over - Seven - holes Hulusi Music}

Known as the Prince of Hulusi Li Chunhua, is China's national first-class actors, performers, composers, educators. Not only in the development of the traditional seven hole Hulusi great contribution, but also in the "super-seven hole Hulusi" spread and promotion played a leading role. Has created the "Son of the Mountain", "Spring Warm Township", "Horse Hulus Variations" and other music, and the preparation of the "eight hole Hulusi basic tutorial" for the "Over seven holes Hulusi" promotion played a role in fueling. Among them, "the son of the mountain" is the first large concerto with the cooperation of Hulusi and symphony in our country. It is of great significance to improve the development and influence of "super-seven hole Hulusi" and the position of Hulusi in the national musical instrument. The range of the traditional gourd instruments on the basis of the up to widen a small three degrees, making the performance of the climax part of the song more in place.

Young Hulusi composer Miao Sheng, under the master of Hulusi art master Lin Rongchang. With the development of eight hole Hulusi, Miao Sheng teacher also created a lot of eight hole Hulusi works, "bamboo morning dawn" "Fen Shui River" by Miao Sheng's younger brother than playing. These works are fully combined with the eight hole Hulusi sound field, showing the rich performance of eight hole Hulusi. There are many young heroes who, in different ways, contribute their own strength to the development of "super-seven-hole gourd".

Famous silk and bamboo musician Qiao Zhichen, is from the famous flute master Liu Guanle, in China's silk and bamboo music in the great contribution, after retirement in his later years focused on Hulusi, developed a number of new techniques Hulusi. In the development of the gourd range, developed the "average law nine hole Hulusi", "to expand the range of eight hole Hulusi" and "low octave of the Hulusi" and the preparation of the "Hulu Si senior teaching counseling Reference "," to expand the range of eight holes Hulusi "two sets of teaching materials, so that we all know, eight holes, nine hole Hulusi. Qiao Zhichen contributed a great development to broaden the development of the gourd range.

As the "super seven hole Hulusi" range of widening, compared to the traditional seven hole Hulusi, "Over seven holes Hulusi" can also play many familiar songs and other musical instruments. Showing a "Over seven holes Hulusi" strong and rich expression. Now in the early stages of the development 
of the "super-seven hole Hulusi" music, the number of solo ensembles for its creation is relatively small.

\section{Communication of Over - Seven - holes Hulusi Music}

"Over seven holes Hulusi" music transmission mainly to Li Chunhua team-based, musical instrument production, music creation, performance practice, lectures and so on, reflecting a series of means of communication. In early 2014, Li Chunhua in Kunming, Yunnan opened the first eight hole Hulusi playing training courses, attracting a large number of "super seven hole Hulusi" enthusiasts to follow; in December 2015 the country's first super seven holes wide range of Hulusi music Will be held in Xiamen, Fujian successfully held the whole evening under the leadership of Li Chunhua, for artists from both sides of the strait to provide a sense of hearing and visual feast; early in $2016 \mathrm{Li}$ Chunhua held in Linyi, Shandong "Over seven holes Hulusi" performance art workshop , In order to "Over seven holes Hulusi" development once again contributed their own strength; 2016 May Li Chunhua held in Changsha, Hunan, "Li Chunhua super seven hole professional gourd workshop" not only to the students brought the performance Greatly improved and there are a lot of touches. They set up a special "new silk rhyme gourd silk art group", and the development of the "Over seven holes Hulusi" WeChat public number, a variety of means to spread.

Other "super seven hole Hulusi" music communication practice, in June 2016 Xiamen Hulusi Association in the summer for the newspaper enthusiastic readers and members of the Association and students held Over seven holes Hulusi public training courses; August 2016 " In 2016, the young Hulusi artist Han Shengli in Inner Mongolia Hulu Sibau art summit forum in the brief description of the advantages and disadvantages of the instrument, in the light of the advantages and disadvantages of the instrument, And demonstrated the "horizon" "Hongyan" and other music.

\section{An Analysis of the Development Prospect of Over - Seven - holes Hulusi}

"Over seven holes Hulusi" is the older generation of artists put forward, modern to Li Chunhua, led by Hulusi artists and Hulusi craftsmen work together results. Throughout the history of the development of Hulusi, Hulusi did not get out of Yunnan ethnic minorities before, almost every person will be blowing Hulusi will produce Hulusi, but the production of the method according to the spread of their fingers with the interval between the standard production Out of the Hulusi has a lot of random, not standardized and standard. And then later through the father of Hulusi all the norms, Hulusi gradually to the standardization and standardization of the road, for the later Hulusi can be popular in the country to lay the foundation.

"Over seven holes Hulusi" from the concept to the real trend to the norms of the slow pace of time there are two. One is the development of society, the emergence of the machine instead of hand-made, so that a lot of Hulusi craftsmen to give up the hand-made, technically no longer more refined study. The other is the production of Hulusi in the course of learning did not form a standardized system, and these Hulusi craftsmen and more dispersed, to discuss the exchange of related technology less. In the development of communication "super seven hole gourd", should be like Hulusi music as the establishment of associations, to discuss each other, for the "super seven hole Hulusi" to mature is bound to have greater help. Hulusi craftsmen should also be with Hulusi composers and performers to actively explore, complement each other and common development.

For the understanding of the super-seven hole Hulusi, due to the short development time, there is no more classic learning tutorial, making many lovers in the learning process has a lot of puzzled and misunderstanding. Mr. Li Chunhua in 2002 published "Hulu Siba practical tutorial" experienced experience and simple and clear language, so many fans do not even have to find a teacher can learn their own good results. But also make the lovers in the process of learning to form a correct understanding of the concept of Hulusi. Which for now "Over seven holes Hulusi" development has a great reference.

Traditional Hulusi in the eyes of many musicians that the range is narrow and too easy to learn and that is the general entertainment toys. The other instruments are less and less, which caused the 
Hulusi can only solo or ensemble, playing together and can not match with other instruments of the embarrassing scenes, and finally make the form of a single Hulusi performance. "Super seven hole Hulusi" appears to make up for the traditional gourd range narrow range, in the future cooperation with other instruments has become a trend in the development. In the orchestra cooperation, the reasons for less cooperation is that the traditional gourd range is not enough to write the song are mostly 3 to 6 minutes, the time is shorter. The appearance of the super-seven-hole Hulusi is an opportunity for Hulu Si to cooperate with the orchestra, and the cooperation with the orchestra can be more rich in Hulusi music, but also can Hulusi to a higher platform.

"Super seven hole Hulusi" to Hulusi music development pointed out a more broad road. Hulusi music is not only an important culture of ethnic minorities in Yunnan, but also an important part of our national culture. Protection and development of super-seven hole Hulusi, not only can increase the position of Hulusi in the national music, but also in the national music to the world, will improve our national music on the world's influence. Super seven hole Hulusi is a turning point in the development of Hulusi, with the social attention and support, the development of super-seven hole Hulusi will be like Hulusi from Yunnan ethnic minority to go out, to a higher platform. For the history of human music add more beautiful symbols.

\section{References}

[1] Qiao Zhichen. Hulusi new techniques and special fingering, Jiangsu: Suzhou University Press, 2014.

[2] Li Chunhua. Eight hole Hulusi basic course, Yunnan: Yunnan Education Press, 2014.

[3] Han Shengli. On the development of seven holes wide range of Hulusi, Minority musical instrument network, 2016,12.

[4] Meng Qi. Over seven holes Hulusi open Hulusi hit a new era, Music Weekly, 2012,1.

[5] Ding Chuanming. Music star - improved porous Hulusi, Yellow River Voice, 2014,6. 